\title{
DUAL PRESENTATION AND LINEAR BASIS OF THE TEMPERLEY-LIEB ALGEBRAS
}

\author{
Eon-Kyung Lee And SAng-Jin LeE
}

\begin{abstract}
The braid group $B_{n}$ maps homomorphically into the Temperley-Lieb algebra $T L_{n}$. It was shown by Zinno that the homomorphic images of simple elements arising from the dual presentation of the braid group $B_{n}$ form a basis for the vector space underlying the Temperley-Lieb algebra $T L_{n}$. In this paper, we establish that there is a dual presentation of Temperley-Lieb algebras that corresponds to the dual presentation of braid groups, and then give a simple geometric proof for Zinno's theorem, using the interpretation of simple elements as non-crossing partitions.
\end{abstract}

\section{Introduction}

Since Jones $[7,8]$ discovered the Jones polynomial for links by investigating representations of braid groups into Hecke algebras and Temperley-Lieb algebras, Temperley-Lieb algebras have played important roles in the quantum invariants of links and 3-manifolds. The Temperley-Lieb algebra $T L_{n}$ is defined on non-invertible generators $e_{1}, \ldots, e_{n-1}$ with the relations:

$$
e_{i} e_{j}=e_{j} e_{i} \text { for }|i-j| \geq 2 ; \quad e_{i}^{2}=e_{i} ; \quad e_{i} e_{i \pm 1} e_{i}=\tau e_{i}
$$

along with a complex number $\tau$ (see $[8,9]$ ). Setting $\tau=1 / \delta^{2}$ and $e_{i}=(1 / \delta) d_{i}$, we get an equivalent presentation, which is easily understood by diagrams, with non-invertible generators $d_{1}, \ldots, d_{n-1}$ and defining relations

$$
d_{i} d_{j}=d_{j} d_{i} \text { for }|i-j| \geq 2 ; \quad d_{i}^{2}=\delta d_{i} ; \quad d_{i} d_{i \pm 1} d_{i}=d_{i}
$$

along with a complex number $\delta$ (see $[10,12])$. It is well-known that the dimension of $T L_{n}$ is the $n$th Catalan number $\mathcal{C}_{n}=\frac{1}{n+1}\left(\begin{array}{c}2 n \\ n\end{array}\right)$. Setting $t$ such that $\tau^{-1}=2+t+t^{-1}$, and then setting $h_{i}=(t+1) e_{i}-1$, we get an alternative presentation of $T L_{n}$ with invertible generators $h_{1}, \ldots, h_{n-1}$ satisfying the

Received April 23, 2006; Revised March 7, 2007.

2000 Mathematics Subject Classification. Primary 20F36; Secondary 57M27.

Key words and phrases. Temperley-Lieb algebra, braid group, dual presentation, noncrossing partition.

This work was supported by the Korea Science and Engineering Foundation (KOSEF) grant funded by the Korea government (MOST) (No. R01-2007-000-20293-0). 
relations:

$$
\begin{aligned}
& h_{i} h_{j}=h_{j} h_{i} \quad \text { if }|i-j| \geq 2 \\
& h_{i} h_{i+1} h_{i}=h_{i+1} h_{i} h_{i+1} \\
& h_{i}^{2}=(t-1) h_{i}+t \\
& h_{i} h_{i+1} h_{i}+h_{i} h_{i+1}+h_{i+1} h_{i}+h_{i}+h_{i+1}+1=0 .
\end{aligned}
$$

The braid group $B_{n}$ is defined by the Artin presentation, where the generators are $\sigma_{1}, \ldots, \sigma_{n-1}$ and the defining relations are

$$
\begin{aligned}
& \sigma_{i} \sigma_{j}=\sigma_{j} \sigma_{i} \quad \text { if }|i-j| \geq 2 \\
& \sigma_{i} \sigma_{i+1} \sigma_{i}=\sigma_{i+1} \sigma_{i} \sigma_{i+1} \quad \text { for } i=1, \ldots, n-2 .
\end{aligned}
$$

The braid group $B_{n}$ maps homomorphically into the Temperley-Lieb algebra $T L_{n}$ under $\pi: \sigma_{i} \mapsto h_{i}$. There is another presentation [4] with generators $a_{j i}$ $(1 \leq i<j \leq n)$ and defining relations

$$
\begin{aligned}
& a_{l k} a_{j i}=a_{j i} a_{l k} \quad \text { if }(l-j)(l-i)(k-j)(k-i)>0 ; \\
& a_{k j} a_{j i}=a_{j i} a_{k i}=a_{k i} a_{k j} \quad \text { for } i<j<k .
\end{aligned}
$$

The generators $a_{j i}$ 's are related to the $\sigma_{i}$ 's by

$$
a_{j i}=\sigma_{j-1} \sigma_{j-2} \cdots \sigma_{i+1} \sigma_{i} \sigma_{i+1}^{-1} \cdots \sigma_{j-2}^{-1} \sigma_{j-1}^{-1} .
$$

Bessis [1] showed that there is a similar presentation, called the dual presentation, for Artin groups of finite Coxeter type.

Both the Artin and dual presentations of the braid group $B_{n}$ determine a Garside monoid, as defined by Dehornoy and Paris [6], where the simple elements play important roles. Nowadays, it becomes more and more popular to describe simple elements arising from the dual presentation via non-crossing partitions. Non-crossing partitions are useful in diverse areas $[1,5,2,3,11]$, because they have beautiful combinatorial structures.

Let $P_{1}, \ldots, P_{n}$ be the points in the complex plane given by $P_{k}=\exp \left(-\frac{2 k \pi}{n} i\right)$. See Figure 1. Recall that a partition of a set is a collection of pairwise disjoint subsets whose union is the entire set. Those subsets (in the collection) are called blocks. A partition of $\left\{P_{1}, \ldots, P_{n}\right\}$ is called a non-crossing partition if the convex hulls of the blocks are pairwise disjoint.

A positive word of the form $a_{i_{1} i_{2}} a_{i_{2} i_{3}} \cdots a_{i_{k-1} i_{k}}, i_{1}>i_{2}>\cdots>i_{k}$, is called a descending cycle and denoted $\left[i_{1}, i_{2}, \ldots, i_{k}\right]$. Two descending cycles $\left[i_{1}, \ldots, i_{k}\right]$ and $\left[j_{1}, \ldots, j_{l}\right]$ are said to be parallel if the convex hulls of $\left\{P_{i_{1}}, \ldots, P_{i_{k}}\right\}$ and of $\left\{P_{j_{1}}, \ldots, P_{j_{l}}\right\}$ are disjoint. The simple elements are the products of parallel descending cycles.

We remark that the definition of simple elements depends on the presentations. For example, the simple elements arising from the Artin presentation are in one-to-one correspondence with permutations. Throughout this note, we consider only the simple elements arising from the dual presentation of braid groups as above. 


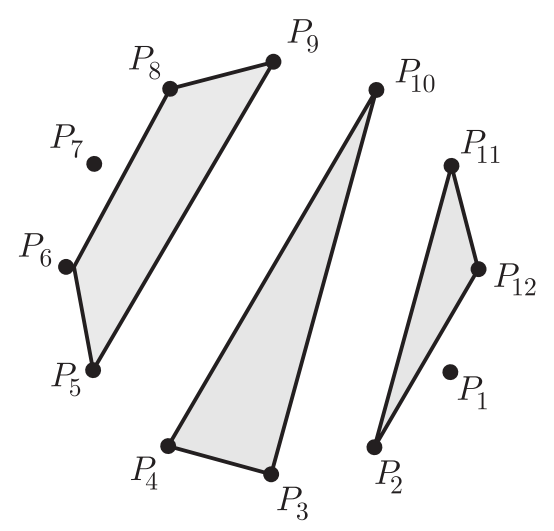

Figure 1. The shaded regions show the blocks in the non-crossing partition corresponding to the simple element $[12,11,2][10,4,3][9,8,6,5]$ in $B_{12}$.

Note that simple elements are in one-to-one correspondence with non-crossing partitions. Our convention is that if a block in a non-crossing partition consists of a single point, then the corresponding descending cycle is the identity (i.e., the descending cycle of length 0). In particular, the number of the simple elements is the $n$th Catalan number $\mathcal{C}_{n}$, which is the dimension of $T L_{n}$. Zinno [13] established the following result.

Theorem 1 (Zinno [13]). The homomorphic images of the simple elements arising from the dual presentation of $B_{n}$ form a linear basis for the TemperleyLieb algebra $T L_{n}$.

We explain briefly Zinno's proof. It is known that the ordered reduced words

$$
\left(h_{j_{1}} h_{j_{1}-1} \cdots h_{k_{1}}\right)\left(h_{j_{2}} h_{j_{2}-1} \cdots h_{k_{2}}\right) \cdots\left(h_{j_{p}} h_{j_{p}-1} \cdots h_{k_{p}}\right),
$$

where $j_{i} \geq k_{i}, j_{i+1}>j_{i}$ and $k_{i+1}>k_{i}$, form a linear basis of $T L_{n}$, and Zinno showed that the matrix for writing the images of simple elements as the linear combination of the ordered reduced words is invertible. Because the number of the simple elements is equal to the dimension of $T L_{n}$, this proves the theorem.

In this note, we first establish that there is a dual presentation of $T L_{n}$. We are grateful to David Bessis for pointing out that the relation (4) in the Temperley-Lieb algebra presentation is equivalent to the forth relation in the dual presentation in the following theorem.

Theorem 2 (Dual presentation of $T L_{n}$ ). The Temperley-Lieb algebra $T L_{n}$ has a presentation with invertible generators $g_{j i}(1 \leq i<j \leq n)$ satisfying the 


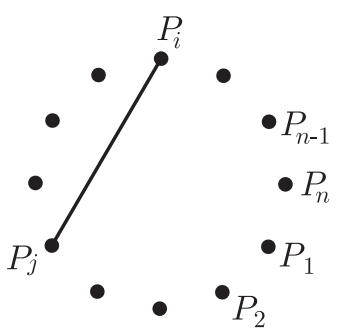

(a)

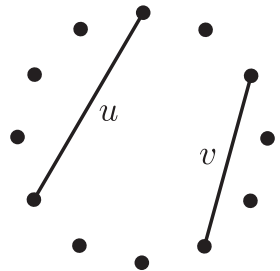

(b)

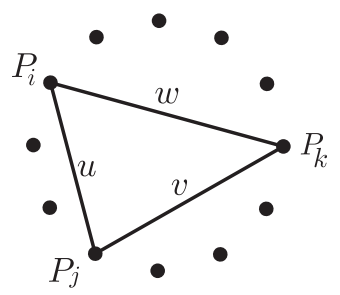

(c)

Figure 2

relations:

$$
\begin{aligned}
& g_{l k} g_{j i}=g_{j i} g_{l k} \quad \text { if }(l-j)(l-i)(k-j)(k-i)>0 ; \\
& g_{k j} g_{j i}=g_{j i} g_{k i}=g_{k i} g_{k j} \quad \text { for } i<j<k ; \\
& g_{j i}^{2}=(t-1) g_{j i}+t \quad \text { for } i<j ; \\
& g_{j i} g_{k j}+t g_{k j} g_{j i}+g_{k j}+g_{j i}+t g_{k i}+1=0 \quad \text { for } i<j<k .
\end{aligned}
$$

The new generators are related to the old ones by

$$
g_{j i}=h_{j-1} h_{j-2} \cdots h_{i+1} h_{i} h_{i+1}^{-1} \cdots h_{j-2}^{-1} h_{j-1}^{-1} .
$$

Using the above presentation, we give a new proof of Zinno's theorem in $\S 3$. We exploit non-crossing partitions so as to make the proof easy and intuitive. For the proof, we show that any monomial in the $h_{i}^{ \pm 1}$ 's can be written as a linear combination of the images of simple elements. Therefore the images of simple elements span $T L_{n}$. As a result, they form a linear basis of $T L_{n}$ because the number of simple elements is equal to the dimension of $T L_{n}$.

We remark that it seems possible to prove the linear independence of the images of the simple elements directly from the relations in the dual presentation of $T L_{n}$ (without using the fact that the dimension of $T L_{n}$ is the same as the number of simple elements), but that would be beyond the scope of this note because it would require repeating all the arguments used in the proof for the embedding of the positive braid monoid in the braid group.

Acknowledgements. We are very grateful to David Bessis for the intensive discussions during his visit to Korea Institute for Advanced Study in June 2003.

\section{Dual presentation of the Temperley-Lieb algebras}

Let $D^{2}$ be the disc in the complex plane with radius 2 and $P_{1}, \ldots, P_{n}$ be the points in $D^{2}$ given by $P_{k}=\exp \left(-\frac{2 k \pi}{n} i\right)$. Let $D_{n}=D^{2} \backslash\left\{P_{1}, \ldots, P_{n}\right\}$. The braid group $B_{n}$ can be regarded as the group of self-homeomorphisms of $D_{n}$ that fix the boundary pointwise, modulo isotopy relative to the boundary. The generators $\sigma_{i}$ and $a_{j i}$ correspond to the positive half Dehn-twists along the arcs $P_{i} P_{i+1}$ and $P_{i} P_{j}$, respectively. 
Let $\mathcal{L}_{n}=\left\{P_{i} P_{j} \mid i \neq j\right\}$ be the set of line segments as in Figure 2 (a). We say that a pair $(u, v) \in \mathcal{L}_{n}^{2}$ is parallel if $u$ and $v$ are disjoint as in Figure 2 (b), and admissible if $u=P_{i} P_{j}$ and $v=P_{j} P_{k}$ for some pairwise distinct points $P_{i}, P_{j}$ and $P_{k}$ which are in counterclockwise order on the unit circle as in Figure 2 (c). A triple $(u, v, w) \in \mathcal{L}_{n}^{3}$ is said to be admissible if so are all the pairs $(u, v),(v, w)$ and $(w, u)$. For $u=P_{i} P_{j}$ with $i<j$, let $a_{u}$ denote the generator $a_{j i}$ of the dual presentation of $B_{n}$. Then the dual presentation of $B_{n}$ can be written as follows:

$$
B_{n}=\left\langle\begin{array}{c|l}
a_{u} & \begin{array}{l}
a_{u} a_{v}=a_{v} a_{u} \quad \text { if } u \text { and } v \text { are parallel } \\
\left(u \in \mathcal{L}_{n}\right)
\end{array} \\
a_{u} a_{v}=a_{v} a_{w}=a_{w} a_{u} \quad \text { if }(u, v, w) \text { is admissible }
\end{array}\right\rangle .
$$

It is easy to see the following: (i) if $u \cap v=\left\{P_{i}\right\}$ for some $P_{i}$ in the unit circle, then exactly one of $(u, v)$ and $(v, u)$ is admissible; (ii) if $(u, v)$ is admissible, then $a_{u} a_{v}$ can be written in three ways as in the presentation, but $a_{v} a_{u}$ is not equivalent to any other positive word on the $a_{u}$ 's; (iii) $a_{u} a_{v}$ is a simple element if and only if $(u, v)$ is parallel or admissible.

Now we prove Theorem 2. For $u=P_{i} P_{j}$ with $i<j$, let $g_{u}$ denote the generator $g_{j i}$ of the presentation in that theorem. Then the presentation can be reformulated as follows. Its proof is elementary. However, we present it for completeness.

Theorem 3 (Dual presentation of $T L_{n}$-reformulated). $T L_{n}$ has a presentation with invertible generators $g_{u}\left(u \in \mathcal{L}_{n}\right)$ satisfying the relations:

$$
\begin{aligned}
& g_{u} g_{v}=g_{v} g_{u} \quad \text { if } u \text { and } v \text { are parallel; } \\
& g_{u} g_{v}=g_{v} g_{w}=g_{w} g_{u} \quad \text { if }(u, v, w) \text { is admissible; } \\
& g_{u}^{2}=(t-1) g_{u}+t \quad \text { for } u \in \mathcal{L}_{n} ; \\
& g_{v} g_{u}+t g_{u} g_{v}+g_{u}+g_{v}+t g_{w}+1=0 \quad \text { if }(u, v, w) \text { is admissible. }
\end{aligned}
$$

Proof. From the results on the dual presentation of $B_{n}$ in [4], it follows that the relations (1) and (2) are equivalent to the relations (5) and (6).

Assume the relations (1), (2), and hence (5), (6).

$(7) \Rightarrow(3) \quad$ It is clear since (3) is a special case of $(7)$.

$(3) \Rightarrow(7) \quad$ It is clear since each $g_{u}$ is conjugate to $h_{i}$ (for some $i$ ) by a monomial in the $h_{j}$ 's.

Now assume the relations (1), (2), (3), and hence (5), (6), (7)

$(8) \Rightarrow(4) \quad$ Let $u=P_{i+2} P_{i+1}, v=P_{i+1} P_{i}$ and $w=P_{i} P_{i+2}$. Then $g_{u}=h_{i+1}$, $g_{v}=h_{i}$ and $(u, v, w)$ is admissible. Since $h_{i} h_{i+1} h_{i}=g_{v} g_{u} g_{v}=g_{v} g_{v} g_{w}=$ $\left((t-1) g_{v}+t\right) g_{w}=(t-1) g_{v} g_{w}+t g_{w}=(t-1) g_{u} g_{v}+t g_{w}$

$$
\begin{aligned}
& h_{i} h_{i+1} h_{i}+h_{i} h_{i+1}+h_{i+1} h_{i}+h_{i}+h_{i+1}+1 \\
= & \left((t-1) g_{u} g_{v}+t g_{w}\right)+g_{v} g_{u}+g_{u} g_{v}+g_{v}+g_{u}+1 \\
= & g_{v} g_{u}+t g_{u} g_{v}+g_{u}+g_{v}+t g_{w}+1=0 .
\end{aligned}
$$


$(4) \Rightarrow(8) \quad$ Note that for each admissible triple $\left(u^{\prime}, v^{\prime}, w^{\prime}\right)$, there is a selfhomeomorphism of $D_{n}$ sending $\left(u^{\prime}, v^{\prime}, w^{\prime}\right)$ to $(u, v, w)$. Therefore, there is a monomial $x$ in the $h_{j}$ 's such that $x g_{u^{\prime}} x^{-1}=g_{u}, x g_{v^{\prime}} x^{-1}=g_{v}$ and $x g_{w^{\prime}} x^{-1}=$ $g_{w}$, simultaneously. Let $u^{\prime}=P_{i+2} P_{i+1}, v^{\prime}=P_{i+1} P_{i}$ and $w^{\prime}=P_{i} P_{i+2}$. In the same way as in $(8) \Rightarrow(4)$, we obtain

$$
\begin{aligned}
& g_{v} g_{u}+t g_{u} g_{v}+g_{u}+g_{v}+t g_{w}+1 \\
= & x\left(g_{v^{\prime}} g_{u^{\prime}}+t g_{u^{\prime}} g_{v^{\prime}}+g_{u^{\prime}}+g_{v^{\prime}}+t g_{w^{\prime}}+1\right) x^{-1} \\
= & x\left(h_{i} h_{i+1} h_{i}+h_{i} h_{i+1}+h_{i+1} h_{i}+h_{i}+h_{i+1}+1\right) x^{-1} \\
= & 0 .
\end{aligned}
$$

\section{A new proof of Zinno's theorem}

Before starting the proof of Zinno's theorem, let us observe the relations $g_{u}^{2}=$ $(t-1) g_{u}+t$ and $g_{v} g_{u}+t g_{u} g_{v}+g_{u}+g_{v}+t g_{w}+1=0$ in the dual presentation of $T L_{n}$. Among the monomials in the relations, all except $g_{u}^{2}$ and $g_{v} g_{u}$ are images of simple elements. Therefore the relations can be interpreted as instructions for converting a product of two generators into a linear combination of the images of simple elements:

$$
\begin{aligned}
g_{u}^{2} & =(t-1) \pi\left(a_{u}\right)+t \\
g_{v} g_{u} & =-t \pi\left(a_{u} a_{v}\right)-\pi\left(a_{u}\right)-\pi\left(a_{v}\right)-t \pi\left(a_{w}\right)-1 .
\end{aligned}
$$

Generalizing this idea, we will show in Proposition 4 that for a simple element $A$ and an Artin generator $\sigma_{i}$, the homomorphic image $\pi\left(A \sigma_{i}\right)$ in $T L_{n}$ can be written as a linear combination of the images of simple elements.

Recall that the simple elements are in one-to-one correspondence with noncrossing partitions. For a simple element $A$, take union of the convex hulls of the blocks in the non-crossing partition of $A$, and then remove those containing only one point. The resulting set is called the underlying space of $A$ and denoted $\bar{A}$.

It is known that for a simple element $A$ and $u \in \mathcal{L}_{n}, A a_{u}$ is a simple element if and only if for any $w \in \mathcal{L}_{n}$ with $w \subset \bar{A}$, the product $a_{w} a_{u}$ is a simple element, in other words, $(w, u)$ is parallel or admissible [4, Corollary 3.6]. Figure 3 shows typical cases of $(\bar{A}, u)$ such that $A a_{u}$ becomes a simple element, and Figure 4 shows some cases of $(\bar{A}, u)$ such that $A a_{u}$ is not a simple element.

It is easy to see that if $\bar{A}$ and $u$ satisfy one of the following conditions, then $A a_{u}$ is a simple element and its underlying space is the union of the convex hulls of components of $\bar{A} \cup u$.

- $\bar{A}$ and $u$ are disjoint.

- $\bar{A}$ and $u$ intersect at the boundary of $u$ as in the left hand sides of Figure 3. Intuitively, when we stand at an intersection point, with $u$ on the right and the component of $\bar{A}$ containing the intersection point on the left, we are facing towards the inside of the unit circle. 

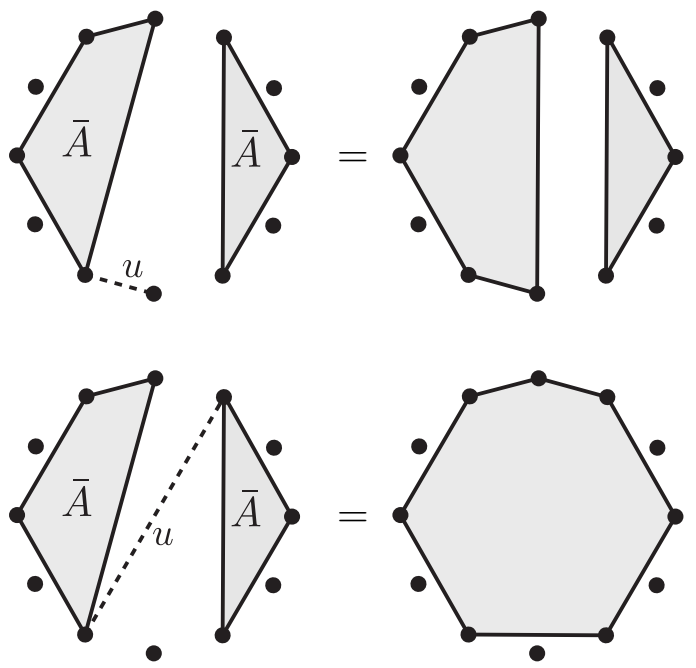

Figure 3 . In the left hand sides $\bar{A}$ and $u$ are depicted as shaded regions and dotted lines. The right hand sides show the underlying spaces of $A a_{u}$ 's.
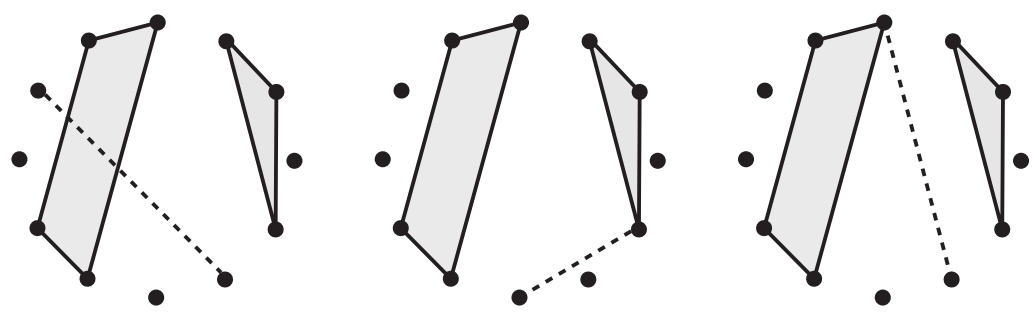

FiguRE 4. The shaded regions and the dotted lines represent the underling space of a simple element $A$ and an element $u \in \mathcal{L}_{n}$, respectively. In this case, $A a_{u}$ is not a simple element.

Proposition 4. For a simple element $A$ and an Artin generator $\sigma_{i}, \pi\left(A \sigma_{i}\right)$ can be expressed as a linear combination of the images of simple elements.

Proof. Let $u=P_{i} P_{i+1}$. Then $\sigma_{i}=a_{u}$ and $\pi\left(\sigma_{i}\right)=g_{u}$. We prove the assertion in three cases.

Case 1. If $u \subset \bar{A}$, then $\bar{A}$ and $u$ are as in Figure 5 (a). Let $B$ be the simple element whose underlying space is as in Figure 5 (b). More precisely, the noncrossing partition of $B$ is obtained from that of $A$ by making $\left\{P_{i}\right\}$ a new block. 


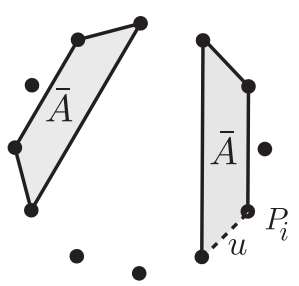

(a)

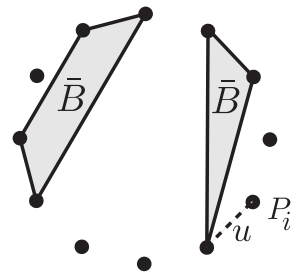

(b)

Figure 5. $A=B a_{u}$ if $\bar{A}, u$ and $\bar{B}$ are as above.
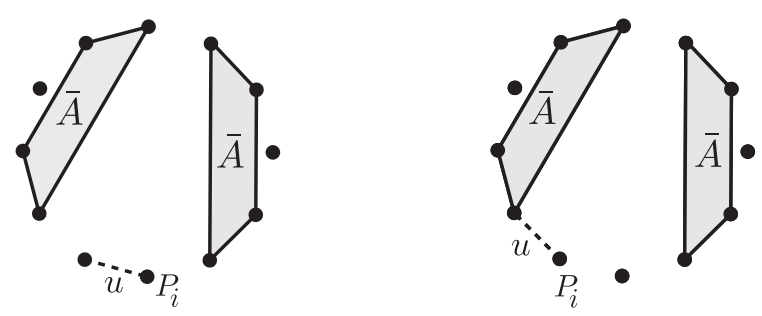

Figure $6 . A a_{u}$ is a simple element.

Then $A=B a_{u}$ and

$$
\begin{aligned}
\pi\left(A a_{u}\right) & =\pi\left(B a_{u}^{2}\right)=\pi(B) g_{u}^{2}=\pi(B)\left((t-1) g_{u}+t\right) \\
& =(t-1) \pi\left(B a_{u}\right)+t \pi(B)=(t-1) \pi(A)+t \pi(B) .
\end{aligned}
$$

Case 2. If $u \not \subset \bar{A}$ and $P_{i} \notin \bar{A}$, then $\bar{A}$ and $u$ are as in Figure 6. In this case, $A a_{u}$ itself is a simple element.

Case 3. If $u \not \subset \bar{A}$ and $P_{i} \in \bar{A}$, then $\bar{A}$ and $u$ are as in either (a) or (b) of Figure 7 , depending on whether $P_{i+1}$ belongs to $\bar{A}$ or not. Let $v$ be the line segment containing $P_{i}$ such that $B a_{v}=A$ for some simple element $B$ as in (c) and (d) of Figure 7. (More precisely, $v=P_{i} P_{j}$ for some $P_{j}$ such that $P_{i} P_{j} \subset \bar{A}$ and the interior of $P_{i+1} P_{j}$ does not intersect $\bar{A}$.) Let $w$ be the line segment connecting the endpoints of $u$ and $v$ other than $P_{i}$. Then $(u, v, w)$ is admissible and

$$
\begin{aligned}
\pi\left(A a_{u}\right) & =\pi\left(B a_{v} a_{u}\right)=\pi(B) g_{v} g_{u} \\
& =-\pi(B)\left(t g_{u} g_{v}+g_{u}+g_{v}+t g_{w}+1\right) \\
& =-t \pi\left(B a_{u} a_{v}\right)-\pi\left(B a_{u}\right)-\pi\left(B a_{v}\right)-t \pi\left(B a_{w}\right)-\pi(B) .
\end{aligned}
$$

Note that $B a_{u} a_{v}, B a_{u}, B a_{v}$ and $B a_{w}$ are simple elements.

Proof of Theorem 1. Let $V_{n}$ be the subspace (of $T L_{n}$ ) spanned by the images of simple elements. Since the number of simple elements is equal to the dimension 


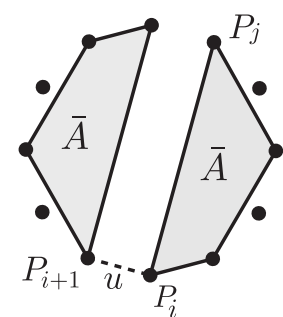

(a)

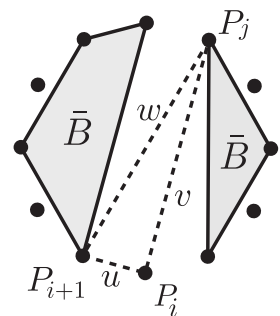

(c)

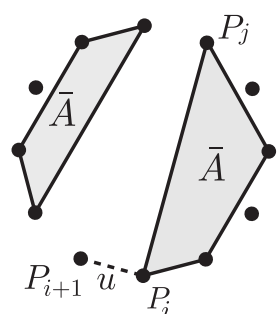

(b)

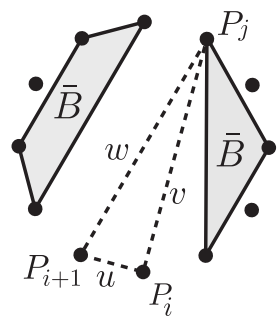

(d)

Figure 7

of $T L_{n}$, the images of simple elements form a linear basis of $T L_{n}$ if we show that $V_{n}=T L_{n}$ (i.e., every monomial in the $h_{i}^{ \pm 1}$ 's belongs to $V_{n}$ ).

Observe that $h_{i}^{-1}=t^{-1} h_{i}+t^{-1}-1$ and $h_{i}=\pi\left(\sigma_{i}\right)$ for all $i$. Therefore, it suffices to show that the images of monomials in the $\sigma_{i}$ 's belong to $V_{n}$. Use induction on the word length of monomials in the $\sigma_{i}$ 's. By Proposition 4 , it is easy to get the desired result.

\section{References}

[1] D. Bessis, The dual braid monoid, Ann. Sci. Ecole Norm. Sup. (4) 36 (2003), no. 5, 647-683.

[2] D. Bessis and R. Corran, Non-crossing partitions of type (e,e,r), Adv. Math. 202 (2006), no. $1,1-49$.

[3] D. Bessis, F. Digne, and J. Michel, Springer theory in braid groups and the Birman-KoLee monoid, Pacific J. Math. 205 (2002), no. 2, 287-309.

44] J. S. Birman, K. H. Ko, and S. J. Lee, A new approach to the word and conjugacy problems in the braid groups, Adv. Math. 139 (1998), no. 2, 322-353.

[5] T. Brady, A partial order on the symmetric group and new $K(\pi, 1)$ 's for the braid groups, Adv. Math. 161 (2001), no. 1, 20-40.

[6] P. Dehornoy and L. Paris, Gaussian groups and Garside groups, two generalisations of Artin groups, Proc. London Math. Soc. (3) 79 (1999), no. 3, 569-604.

[7] V. F. R. Jones, Index for subfactors, Invent. Math. 72 (1983), no. 1, 1-25.

[8] _ Hecke algebra representations of braid groups and link polynomials, Ann. of Math. (2) 126 (1987), no. 2, 335-388.

[9] L. Kauffman, Knots and Physics, World Scientific, Singapore, 1993. 
[10] W. B. R. Lickorish, Three-manifolds and the Temperley-Lieb algebra, Math. Ann. 290 (1991), no. 4, 657-670.

[11] J. McCammond, Noncrossing partitions in surprising locations, Amer. Math. Monthly 113 (2006), no. 7, 598-610.

[12] V. G. Turaev, Quantum Invariants of Knots and 3-manifolds, de Gruyter Studies in Mathematics, 18. Walter de Gruyter \& Co., Berlin, 1994.

[13] M. G. Zinno, A Temperley-Lieb basis coming from the braid group, J. Knot Theory Ramifications 11 (2002), no. 4, 575-599.

EOn-Kyung LeE

Department of Mathematics

SEJONG University

SeOul 143-747, Korea

E-mail address: eonkyung@sejong.ac.kr

SANG-Jin LeE

Department of Mathematics

KONKUK UNIVERSiTy

Seoul 143-701, Korea

E-mail address: sangjin@konkuk.ac.kr 